\title{
13 The rhetoric and politics of standardization: Measurements and needs for precision
}

Important then to escape the pitfalls of any arbitrariness in understanding what we are doing in counting units of length, weight, or any measurements of areas of space, we need to understand what constitutes the unit of measurement in order to use it practically in what we do when we use measuring tools. Measurements such as sums of units of length, area, of volume, angles, weight, and even sums of units of time are countable. In turn we use these measurements to relate to other things that we also define as being countable.

How we relate to those countable applications depends upon the measuring tools we create. And what constitutes a unit of measurement is established with these measuring tools within the legal frameworks of what might be considered political power or law, whether laws of the tribe or the sovereign power of a nation. As experts we make political demands that become commands of those that have the political power to standardize measurements in manufacturing, construction, trade, and commerce. They create the law that underpins the technical definitional agreements that are made about measurements by those different social institutional agreements made between social corporate structures and government agencies.

Measurements are always made then within certain social and political frameworks. The definitions of measurable units are usually initiated within the jurisdictions of technological and scientific institutions which with their persuasive power are able to eventually reach their legitimized agreements about standards, and they gain their approvable within law using enforceable legal contracts. Thus, units of measurement for the most part actually have a legal basis such that the technical agreements of interest groups are enacted into laws that lay the foundation of any contractual agreement between those using them.

Let me first illustrate a linear model of the metric system (SI) as standardized and defined internationally by agreement and by political consensus. Next let us compare metric system (SI) with the standardizations of the Imperial/American System established by countries that legally adopt them.

\subsection{The Standardization of Metric Measurements}

A meter has been officially defined to be the distance traveled by light in a vacuum in 1/299,792.458 second. All other length and distance measurements in the metric system derive from the meter. 
Table 13.1: Metric Unit Conversion

\begin{tabular}{ll}
\hline Unit & Relation to base \\
\hline kilometer & $10^{3}$ metres \\
hectometer & $10^{2}$ metres \\
decametre & $10^{1}$ metres \\
decimeter & $10^{-1}$ metres \\
centimeter & $10^{-2}$ metres \\
millimeter & $10^{-3}$ metres \\
micrometer & $10^{-6}$ metres \\
nanometer & $10^{-9}$ metres \\
\hline Imperial/American Measurements
\end{tabular}

Note the lack of simplicity in converting various units of measures of length in Imperial/American Measurement System. The difficulty lies in the complexity of the conversions and comparisons of units. A yard can be defined as the length of a pendulum that causes its arc to swing in exactly 1 second. Note the nautical mile is the distance across 1' (1/60 of a degree) around the earth's surface. To simplify calculations the practical rule is to transfer Imperial measurements to the metric system when doing complex measurement problems

The metric system makes visible the relative sizes of the comparisons. Note the following model Figure 13.1 using the metric system of measurement illustrates the relative comparative measurable scale of small units of life and units of matter starting with the larger protozoa largest in size and going down the scale to the somewhat smaller eukaryotes that were the progenitors of all muti-cellular life. These units are compared again in size then with the smaller prokaryotes, and then down to the virus and then to the smaller protein molecules, and finally down to the theoretical estimated size of atoms. Note the illustrating model does not extend further down in scale to the theoretical size of nuclear particles. It is through the use of such numerical scalar models that we are able to make relative comparisons in using models and graphs to think of the scale of things and to make relative comparisons more visual perspicuous. 

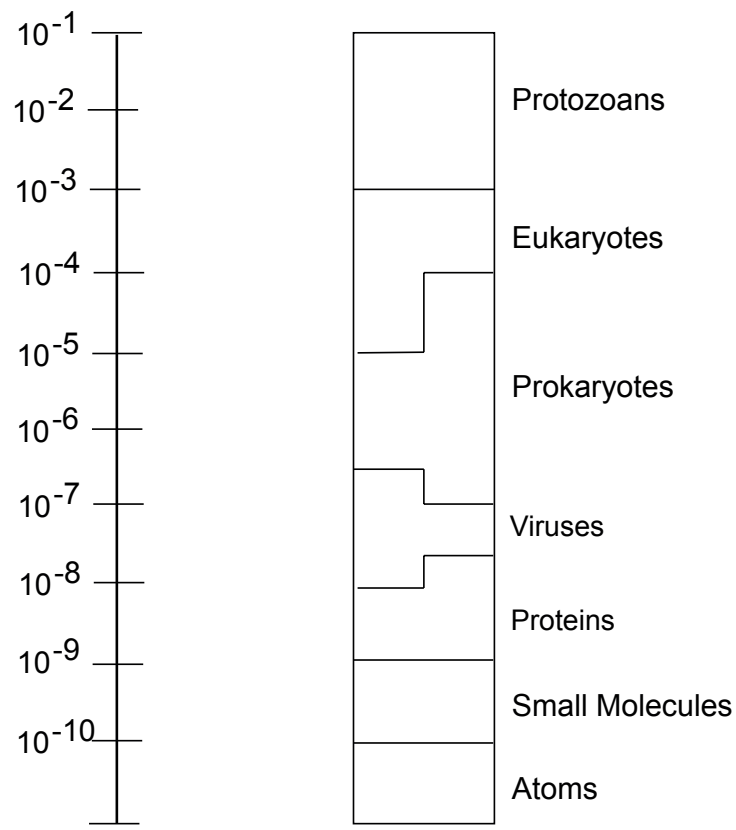

Figure 13.1: Descending Relative Scale of Protazoans, Eukaryotes, Prokaryotes, Viruses, Proteins and Atoms

But note too that the politics and the social politics of measurement have social problems and conflicts. Difficulties arise about the precision and the practical applicability of these legally developed and institutionally technically defined agreed upon standards of measurements. They are issues over values and political power. It is at this point that we see the importance of understanding the rhetoric and the politics of these disputes that take place over the accuracy and the precision of the technical definitions of measurements and the choices that were made in deciding on the standards to be used in differently defined units of measurement. Such negotiations over legalizing the definition of units of measurements present all the measuring complications that create problems between different societies who have their own cultural preferred different accepted standards of measurement to meet their social and political needs.

Difficulties thus arise about the precision and practical applicability of theselegally and institutionally defined technically agreed upon standards of measurements. It is at this point that we see the importance of understanding the rhetoric and the politics of these disputes that take place over the technical definitions of measurements and the choices that we make deciding between the different standards that are used to distinguish between the different applications of these defined units of measurement. It presents all the problems of metric conversion that different societies have who 
still prefer their own cultural standards of measurement over the uses of the metric system. Note the difficulty the United States has in eliminating the penny as a unit of measurement of money. It creates and economy where direct currency exchanges are time consuming. Note how electronic transactions and accounting calculation have eliminated the physical efforts expended in the exchange of worthless pennies.

Note the politics and the history of defining the angstrom unit of light used in spectroscopy:

\begin{abstract}
Although intended to correspond to $1 \times 10^{-10}$ metres, for precise spectral analysis the ångström needed to be defined more accurately than with the use of the metre which until 1960 was still defined based on the length of a bar of metal held in Paris. In 1907 the International Astronomical Union defined the international annsström by declaring the wavelength of the red line of cadmium in air equal to 6438.46963 international ångströms, and this definition was endorsed by the International Bureau of Weights and Measures in 1927. From 1927 to 1960, the ångström remained a secondary unit of length for use in spectroscopy, defined separately from the metre, but in 1960, the length unit metre itself was redefined in spectroscopic terms, thus aligning the ångström as a submultiple of the metre. In short, one nanometre is equal to ten ångströms. Today the use of the ångström as a unit is less popular than it used to be, and the nanometre is often used instead. The ångström is officially discouraged by the International Committee for Weights and Measures. (Oxford English Dictionary)
\end{abstract}

To apply units of measurement then I suggest that we need first to hypothesize about what we directly perceived to be a unit when we try to count anything. We create units literally by taking steps in our thinking to produce or to isolate them. Acts of measurement are based upon our notion that units have boundaries or frames which determine whether something can be perceived as a whole and that such wholes are repeatable and thought identical.

Measurements also involve counting such as we do with the digits of our fingers. We see each finger as a whole and the others like it are also unit appendages of the hand. We line them up to make them separate by contrast. Numbers in one sense then are units or digits that we conceive as counts that we make when we add up our fingers. Primitively counting involved making scratches just as archeologists have found in the early developments of writing. In its elementary form writing had in its very beginning in scratches, which we see in the root historical sense of what it was to write. It first started as a tally of counts. Writers were 'scratchers'. Such was the beginning in the tallying of digits. It was just juxtaposing scratches as symbols of groups of tallies. A count then is based upon combining one unit with another to make an aggregate unit which allows us to keep adding digits by adding each digit to make a new number which we then think of as a new unit, or a tally. One added to one creates a new unit of two, which is a pair, and a pair is a unit as we do in our treating a couple as a unit. And adding a new digit of one to the new unit of two or a pair makes a new unit of three, a trinity, a triple, or a triad. Note the Nicean Creed thinks of the trinity as one. Thus we generate in name a series of new units when we add to each of 
the previous named aggregate or tally. Thus, in sum we can continually create a new unit by continually adding a new unit or a digit to a previously created digit.

I learned from my four year old son very late in my life with no help from any teacher that adding numbers is simply counting up one number the number of times of the value of the other. And also that subtraction is counting down the number of times the value of the other. And multiplication is no more than counting equal tallies and then counting up by the number of the tally by the number of the tallies. And division is just the reverse of that, which explains why in dividing we are always subtracting from the number that we are dividing. That I should have to find this way of dealing with numbers, which is so obvious to any mathematician, illustrates to me the deficiency of my early parochial school training in learning math.

And to continue in thinking this way it occurred to me also that not only do we count with our hands, with the thumb summing up the tally of five with the closure of our thumb, but that by using both hands we generate our decimal system of numbers by using our hands to create two tallies of five. A hand is a set or tally of additions with its five digits. And the number of hands is a count of the tallies of five which is simply adding hands of fingers by a count that we call multiplication.

We see these processes modeled in the design of an abacus. See the sketch of a Chinese abacus in Figure 13.2 below. This historical account and the picture are a copy of a Chinese organization that sells advertising tours and materials that display Chinese culture.

There are five beads to count the digits and two beads to count two tallies of five digits. And a second row of five beads and two more beads to tally the tallies in the first row. And thus the abacus is aligned by rows of rows to keep tallying our arithmetic operations just as we use an order of prefixs to tally up 10's in our decimal system. It is the model of our first mechanical calculator. The abacus is sometimes called a counting frame. It is a calculating tool still used in parts of Asia for doing arithmetic.

But there are different elemental uses of numbers beside our counting or adding by addition or by multiplication by adding tallies. There is the ranking and ordering of digits. Ranking and ordering produce quite different type of models than that illustrated by the abacus. Looking at the palm of our left hand the index finger is left next to the middle finger, the middle finger is left and next to the ring finger, and the ring finger is left and next to our pinky or the little finger.

By defining numbers next to each other, not only are we able to count them, but we are able to order them as well. Thus, we create the distinction between cardinal numbers and ordinal numbers. One counts, and the other ranks or orders. Note the importance of thinking of numbers in a definite defined order. First, second, third, who likes to be third? And if you come in fourth in most races, it does not even count. You do not even show. Note that if something does not count, it never matters. Such are the uses of terms surrounding counting and ordering to rank what we think of things as having importance. 


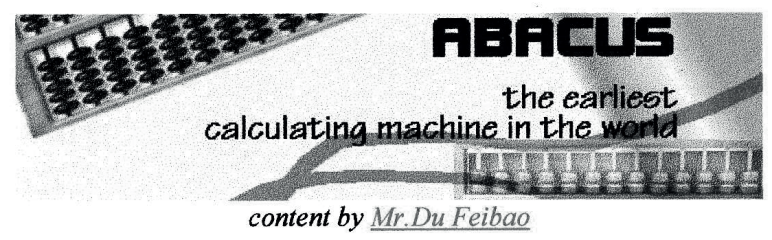

The abacus was a great invention in ancient China and has been called by some Western writers "the earliest calculating machine in the world."

The abacus has a long history behind it. It was already mentioned in a book of the Eastern Han Dynasty, namely Supplementary Notes on the Art of Figures written by Xu Yue about the year $190 \mathrm{~A}$. D. Its popularization occurred at the latest during the Song Dynasty (9601127), when Zhang Zeduan painted his Riverside Scenes at Qingming Festival. In this famous long scroll, an abacus is clearly seen lying beside an account book and doctor's prescriptions on the counter of an apothecary's. During the Ming Dynasty (1368-1644), the abacus was introduced into Japan.

Abacuses are easy to make, handy to carry around and quick to give the answers, provided one knows how to move the beads. They have been in use, therefore, down to this day. They are made in different sizes, and the largest known abacus, measuring 26 centimetres high by 306 centimetres long with 117 rods (for as many digits), is over a hundred years old and is kept at Darentang, a well-known traditional pharmacy in Tianjin.

The beads on an abacus may be round or rhombus in shape. Traditionally, there are two beads above the horizontal bar and five below. Simplified modern versions have one bead above and four or five below. The methods of calculation remain unchanged.

At a time when the world has entered the age of electronics, the abacus still enjoys undiminished vitality in China. Tests have shown that, for operations of addition and subtraction, the abacus is still faster than the electronic calculator. China developed in 1980 an "electronic abacus " which combines the speed of traditional addition and subtraction methods with those of the modern calculator at multiplication and division. It is a happy example of the integration between the East and West, the native and the modern.

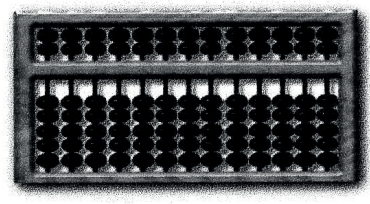

Chinese abacus

Figure 13.2: Chinese Abacus

Units can be counted and ordered. How then do we then recognize or determine a count? Where do we start? How do we describe any order? Temporally we measure time by the regularity of repetition as Galileo supposedly did when he used his pulse to compare the various units of length of a pendulum with the presumed regularity of his pulse. Time in a primitive sense of the term is measured by the number of times that something presumptively regularly repeats itself. 
Primitively then we can use our pulse assuming it is regular for short periods of time. But of course we know our pulse rate changes with any increased bodily activity. Thus time requires that we test the regularity of other repetitive events to determine which repetition of the two is more regular or more precise than the other. We can do that by numerous comparisons and correlations with other presumed seemingly regular repetitive pulsing or beating mechanisms or cycling events. Note the waves moving over the surface of lakes and seas when it is not gusting and blustery come in regularly. Such is the notion of regularity that we establish in music. Elementary is the use of the metronome to determine what is a constant rhythm or a beat. Note the musical conductor is a living metronome, who can change any speed of the beat by gesturing faster or slower, thus regulating and 'synchronizing' the beat of the orchestra. The same holds true in military drill. The drill sergeant establishes the cadence or the beat. 'Hup, two, hip, four!'

But note how musicians can defy our expectation by playing an unanticipated beat. Even as in poetry or song there is rhythm that is fundamentally ordered and based on counts of a beat. But the rhythms of language mostly result from our pacing our words in time as we do in our oral interpretation of poetry. But note that for the most part syllabication and words tend to vary in length and in strength with intonation. It is these spoken separate units in poetry that form a rhythm or a beat, or rhythmically interesting variations on a beat.

As Crystal illustrates the origins of language in a growing child starts with intonation and rhythm. Such as stated is the basis of poetry with its rhythm and its numbered beat and its intonations. We can even measure time by the repetition of the rhythm of a set of repeated words. When refereeing a basketball game, as I did years ago, I learned to count one thousand one, one thousand two, one thousand three, that is, the 3 second count in basketball to keep players from posting up too long under the basket in the area at the bottom of the key.

Spatially we usually measure distance or length by our number of steps or strides. We need to avoid shortening or lengthening our stride to make certain that our steps are uniform. Thus, we can pace off a distance. But we can instead of using as a measure a step just mark off the length of our steps with a measuring stick, which serves then as a more refined or precise unit of measurement. But then linear measurement depends on whose measuring stick that we use, a yard stick or a meter stick, or a rod that is $16 \frac{1}{2}$ feet used for a measuring length in surveying. Note the units that are adopted depend upon what is being measured, the purposes of the measurement, and the demands of the situation.

The ancient Greeks as mentioned first did it with a straight edge and a compass in their geometry. Note the importance of a taut string as a technical procedure to define both a straight edge and a compass. Note then that there are two basic presumptions in geometric constructions in Greek geometry:

1. a straight edge is straight,

2. the compass always draws equal radii when drawing circles. 
The very beginning of the construction of lines and figures begins from these static presumptions behind the use of these two primitive instruments. What is most obvious is that Greek geometry in its constructions first focused on circles, triangles, and squares which are not treated numerically but as areas enclosed within lines. Using Figure 13.3 below let me interpret this elementary proto-geometry using elementary modes of construction as the Greeks approached it.

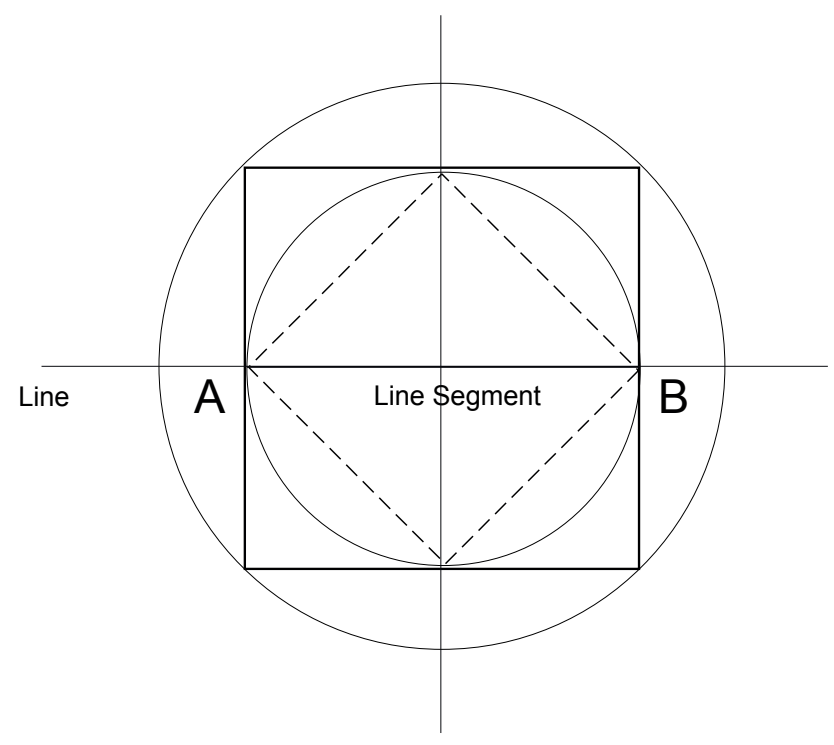

Figure 13.3: Elementary Proto-geometry

Note that when you draw a straight line with a straight edge it is presumed to be straight and when you arbitrarily mark off two points on the straight line using any arbitrary radius of your compass starting from any arbitrary chosen point on that line, such as A, you have by definition marked the end points of line A and B, that is equal distance with the span of the compass. And when you with your compass or divider make the arcs of two larger circles from each of the ends of your line segment, one above and one below from the end points $\mathrm{A}$ and $\mathrm{B}$, you create two points of intersection that allows you to draw a line segment $\mathrm{CD}$ that passes through line segment $\mathrm{AB}$. The points on the intersection of the line segment $C D$ connecting the two intersecting arcs drawn with a compass is said to be the perpendicular bisector of the arbitrarily marked segment of the line AB. Note that this construction of a perpendicular bisector works on a line, on an arc of a circle, or any other smooth curve.

This construction using a straight line and a line between two points on compass or a string as having the same length seems to be the very basic axiom on which all Greek geometry was based. Such was the beginning of all the interesting constructions 
of all the different figures that Greek geometers constructed with the straight edge and the compass, and with which they were able to compare the ratios between all the lines, shapes, and volumes in determinate deductive ways. Such was the way Greeks drew circles and used lines to draw linear closed figures with areas both inscribed and circumscribed. Note especially the affinities of squares and circles in the construction of figures.

Much of our rhetoric to express direction and orientation centers on the three basic simplistic ways we have of constructing three figures: triangles, squares, and circles. They are the basic simple patterns of the models that we have when we think about what we say. We circle around a point. We divide our talking points and our concepts in twos and threes. And we think of triangles combining to form quadrilaterals. When we count four sides we need to think of four as two pairs. Four I suggest then is the beginning of our conceptions of complexity that begin to develop when we get beyond structures of triangles.

In one ordinary sense of the term number, when we speak of one, two, or three they are not considered a number of things. You do not say that there was a number at the party when there were only two or three. And if we think of numbers as describing complexity, that is, a number of things, we need to develop numbers to count the scope of that complexity, thus the rhetorical necessity of measuring by counting to come to terms with the complexity of a number of things.

Quantification in ordinary ways of speaking is both something we communicate by vague estimations or by rigorous enumeration. The weakness of the classical logical syllogism is in the lack of precision in talking about how many and how much and to what degree something is the case. In Medieval logic some could mean at least one, a few, many, most, and not all. Such classical logical quantifications are extremely loose and vague when we speak of 'some' in ordinary discourse.

Let me introduce to you the simple Greek way whereby we can construct an endless string of figures simply by constructing figures out of lines and triangles, circles, tetrahedrons, cubes, cones, pyramids, cylinders, and spheres. What this methodology of construction illustrates is our ability to name and create patterns that prior to the Greeks that few, or maybe no one had created or graphically displayed before. It shows how by rule or by deduction from basic rules and axioms, that we can innovatively create new patterns and forms especially in the field of mathematics.

As has been the case in the development of mathematics many mathematicians devised patterns that others were able then to use further to innovate and discover new patterns for themselves. It is much the same in chess. Players in following the moves made, and in using the logic of chess encounter positions probably never seen before. And chess players if they wish can by description of their positions of their pieces give it a new name, which others then in turn can use to identify the same defined positions in using 'standard chess notation'.

To illustrate the nominal deductive nature of plane or area geometry, let me show by example how there have been traditional proofs without numbers to prove one of 
the most basic theorems of geometry, namely the Pythagorean Theorem, which in its origins was about the areas of square figures constructed using an edge on each side of a right triangle. There are many different ways to prove this theorem, but the following Figure 13.4 demonstrates one simple geometric way without the use of any proofs using numerical algebra.

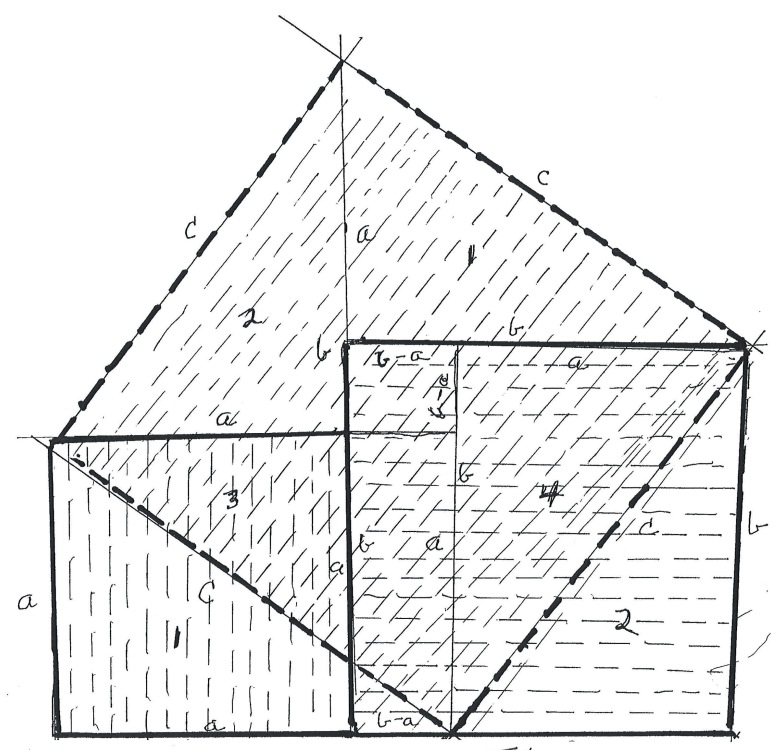

Figure 13.4: Pythagorean Theorem

Given any square with edges c - which is the hypotenuse - and with any rectangle whose area is ( $\mathrm{a} x \mathrm{~b}$ ) divided along its diagonal with edges $\mathrm{a}, \mathrm{b}$, and $\mathrm{c}$ to form similar triangles 1, 2, 3, and 4, we can then construct the square with edges c. In doing so we leave open an uncovered little square area in the center with edges ( $b-a)$. When we add triangles 1 and 2 opposite the figure to 3 and 4 respectively we can by subtracting and adding the rectangle of edge $(b-a)$ and the edge $b$, we can construct square ( $a$ $x a)$ and the square $\{(b-a)+a\}$ or $(b x b)$. Thus we have geometrically covered the empty square with edges ( $b-a)$, thus showing that the square of a and the square of $\mathrm{b}$ are equal in area to the area of the square with the side c. Note our additions and subtractions are of areas and not of numbers. Thus, we visualize geometrically the Pythagorean Theorem in terms of areas not numbers.

However to treat the Pythagorean Theorem numerically algebraically we can illustrate the basic equations give using again Figure 13.4. What results is a simple numerical proof treating a, b, and c as numerical measures of length. From 13.4 we see that the following equation holds: 


$$
\begin{aligned}
& \text { Area of right triangle }=\left(\frac{1}{2}\right) a b \\
& c^{2}=\left[4\left(\frac{1}{2}\right) a b\right]+(b-a)^{2} \\
& a^{2}+b^{2}=c^{2}
\end{aligned}
$$

Thus we can show from the above equation the Pythagorean Theorem expressed numerically as the measured lengths of the hypotenuse and the other two sides.

$$
\begin{aligned}
& c^{2}=2 a b+b^{2}-2 a b+a^{2} \\
& c^{2}=a^{2}+b^{2}
\end{aligned}
$$

Note then the Greeks did not conceive of their linear segments as numbers, but as having ratios and proportions. This leads to an interesting sense of what the Greeks thought of as a number. As already mentioned in ordinary English when we speak of a number of people we do not ordinarily think of one person as constituting a number of people, nor do we even count two as a number of people. Two people together are a couple such as you and me. We two are not a number in the sense of a multitude of people. And when we come to triads it is interesting that three people, you and me and some other person really are not a multitude or a number of people. We are a couple and another person added together. We form a single triad. Exploring love triangles is a major structure in romantic literature.

Triads are simple configurations that are basic to human intuitions and human reason especially in the role taking as we see reflected in syntax and grammar as first, second, and third person. So three people simply reflects how in communication the way we talk in first, in second, and in third person. Note that G. H. Mead's major contribution in Mind Self and Society to social psychology was role taking. There are three that we take: one of self, one of another, and one of that role of that other person outside the self. It is only at four that we need to count what a number of people would be considered as we get outside these basic three roles. It would be two twos, or one against three. $2+2=4$ or $1+3=4$.

Counting seems then to be a way of dealing with what might be described as a number of things, which is the beginning of our thoughts about increasing complexity. With two people we have just one pair or set of a relationship, one to one and one to the other. With three people we have only three sets of relationships, but when we come to four we raise the number of relationships to six. And then if we relate the three and four persons asymmetrically, we see we double the number of relationships. It is only then when we reach four that we are confronted in the beginning with a set of multitudinous relationships, which eventually in time leads to too much complexity to grasp in our thinking.

Note how we think of the number of stars as an image of complexity. Who normally thinks ever to be able to count them? And when you speak of crowds of millions, the population of cities, or the casualties of war we have little capacity to 
image such a large number. It is for that reason that when we consider visible stars as almost enumerable we have made no count. Thus, I was surprised to find out that 'the star catalogue compiled by Claudius Ptolemy in the 2nd century CE lists 1,022 fixed stars visible from Alexandria. This became the standard number of stars in Western culture for hundreds of years. The total number of stars visible to the naked eye is about 6,000; only about half are visible at a given time of night from a given point on the Earth. (Wikipedia). Note our image of the number of stars poetically in not a very big number.

I have tried to illustrate this problem of complexity in dealing with numbers by showing that we rhetorically ordinarily have no clear concepts of the relationships between people in a group when the number in the group has a count of four or more (Yoos, 2009). Clarity in numerosity requires that we have better simplified conceptions and clearer pictures of multitudes. Experiences of numbers require pictures of large groups. Note how difficult it is to estimate the numbers of people in large crowds unless there is some sort of correlation with a number that correlates with a numbered grid such as the number of seats that are filled or empty in an auditorium or maybe how many people occupy an area which we can estimate by the count of such gridded areas that are occupied by people. To be crowded is to be in too dense of a crowd. Note how my terms in talking about crowds circle around proximate meanings and approximations. It is all about finding clearer verbal conceptions about complexity

And it is this reduction of counting to a simple or clearer picture of a number that we initially used the digits of our fingers and our two hands to count either by tallies of fives, or by tallies of tens. Thus it is through digits and by their one to one correspondences between our finger tallies that items can be simplified in counting. We have more familiarity with the pattern of the fingers of our hand than with patterns that we are not so familiar with, such as being together with ten unfamiliar people. It is no accident then that the word digit applies both to fingers and the counting of cardinal numbers that are used for counting.

And consequently as importantly mentioned the use of our two hands in counting becomes a frame also to create order as we can order our fingers in sequence: Left thumb, left index finger, left middle finger, left ring finger, and left pinky. This pattern is equally repeated in the names of the fingers of the right hand. Note this pattern is the decimal system that maps the pattern of the decimal numbers. If human beings had had twelve fingers and twelve toes we probably would have had a different number system, first either to the base twelve or by adding toes to the base twentyfour, one with two tallies of six for each hand, or four tallies of six for all our fingers and toes. And thus it was in such counting that units of measurements began: yards of cloth from the stretch of the arms, hands to measure the height of a horse, and feet to measure the distances around enclosures and the areas of enclosures as we do in talking about the size of houses in terms of square feet. Note how inadequate all these measures have become. 
Thus, the concept of measurement presumes a unit that is rigid and capable of designation. Rigid designators are instruments that provide us with regulated measures of units. And each designation determines a count. And counts are a matter of digits. As we have seen that in mathematics and trigonometry we have both linear and angular measurements, and that both notions of measurement are developed on the basis of digits or counts. When we measure distance we need a concept of something being straight on a line of sight stretching between two points.

When I was in the air corps we were told in navigating that straight lines were man-made. But of course they were talking about lines in a plane. But there are few lines in nature that are straight in all three dimensions. Trees especially bamboo can grow straight and tall. They grow vertically straight when crowded for lack of light being shaded by the surrounding trees. As such straight lines except for trees and bamboo poles are all artifacts with straight lines of man-made construction. But how then do we ever construct straight lines in a plane? They require two points in a plane, but the problem then is 'Are they in the same plane?'

What has fascinated me of late is a Möbius strip and the lack of any concept of orientation on its surface. The Möbius strip is created by reversing the end of a lengthy flat strip of let us say paper, with straight and parallel sides and then paste-ing the reverse ends of it together. What is created is a surface that slowly curves away from any notion of straight lines. What creates my fascination with this construction is that surface of the strip is the same surface on both sides of the strip. If we follow a line moving forward seemingly parallel to the edges of the strip we find that there is no top or bottom to the surface of the strip, for if we mark a spot opposite on the strip we will eventually reach it by moving along the surface of the strip.

What is seemingly opposite to the starting point on the strip is on the same surface that we are located. In other words there is no directionality on the surface such that it has another side. There is no orientation for a line of sight as there is no conception of a straight line on the strip. In other words the Möbius strip displays the relativity of our concepts about what is nominally a plane or a surface. The Möbius strip is a surface with only one side and only one boundary component. The Möbius strip has the mathematical property of being non-orientable. It contains no straight lines or flat surfaces, in that when we construct it, it exists in three dimensions.

A plane is supposedly flat. But we need to determine if something is flat by a level, and we need to recognize that levels and plumb bobs as tools are based on gravitational pull, and thus we know gravitational fields of equal points of field potential are not flat or straight, but they are centered in a circle surrounding a circular mass of earth. All this suggests that what is straight is a concept that is context dependent. If we fly an air plane for instance using an altimeter that is based upon a measure of air pressure. The plane in flying over a period of time is not strictly flying a straight line but an arc, which is due to its flying around the curvature of the earth in air of equal pressure. And if the plane is flying the shortest distance it is not flying a straight compass heading, for the shortest distance on the earth's surface is 
a great circle path which requires constantly changing compass headings to follow it. What is a line of sight or a shortest path again is a matter of context and situation.

It is easy to construct a circle; for by definition or by design by strict definition, a circle is a line in a plane that is equal distance from a point. We use a divider to establish an arbitrary unit on a line; and since the divider establishes rigidly a unit of distance between two points, we can rotate that unit of a straight line in a plane to make a circle. But what determines that the point is equidistant is the assumption that an inelastic string or rod rotates around a point is rigid and at a fixed distance. The assumption in using a divider is that we are measuring a straight line. But what is this assumption based upon? It is always questionable whether a surface is flat and our line is rigidly determined.

That assumption of being flat rests upon the notion that two points determine a line and three points determine a plane, and as for three dimensions that depends on four points not being in the same plane. Thus, what is straight and what is circular depend on how we rotate or turn in any direction, whether we are at a fixed point looking at something in a plane or whether we are looking at a point on a circle. It again is based upon assumptions. It is a determination made from a stationary or stable perspective at the point or the axis of rotation.

But how do we ever determine whether a point is fixed or not. The presumption in measurement is that there is a 'here-now' as a point of reference and we can determine a 'there-then' from a rigid designation of an instrument that uses a defined unit of measurement. There must be a constant rotation or a repeated effort using a rigid rod to determine the difference between 'now and then' and between 'here and there'. And that determination must always be a calculation determined from a fixed point of reference.

Such a status of fixity and any change from it presumes that all measurements of time and space are relative to perspective. Such presumptions are at the heart of all modeling and mapping. We used the so called 'fixed stars' to say that time and space were not at all relative, but they were fixed from the perspective of the celestial observer in the ancient world. But there was no measurable conception of distance to the stars held by the ancients. Planets were heavenly bodies moving in fixed patterns found in the mapping of the fixed stars as reference points.

But the Copernican theory changed the whole perspective. It changed what was the fixed point reference of the individual in relation to the stars. What is up was changed with the notion that the earth was no longer the center of reference. And now we have the same problem of reference in modeling the universe since the sun is no longer fixed, nor is the Milky Way as a galaxy fixed, but it is finding its own merry way, assumed to be widening out supposedly with an expanding universe on the way out to somewhere, but out of what spot towards what? There is no way then of looking up and down except in the context of looking at the universe. Note that astronauts have a sense of what is up in their space vehicles if the vehicle has a frame that has a top and a bottom. Note too that what we read gives us a sense of up, for we know 
that up is at the top of the page. The ancient civilizations developed geometry as earth measurement out of ordinary constructions of lines and their orientations to develop a way of defining boundaries to their land and arable fields. One needs a fixed point of reference to start drawing a line with an orientation to another fixed line assuming that we are on a level plain.

When I first studied Euclid we studied it not algebraically, but with a straight edge and a compass. And the first step in our construction of figures and circles as I have already illustrated was to learn to divide a unit of a line, seemingly a straight line, between two points by learning to draw a perpendicular bisector. To do that required us to draw arcs of a circle with a compass from the two different points on the line. What struck me in doing this is that a perpendicular bisector presumed that the line drawn from the intersection of the two arcs above and below the line did in fact create four right angles around the point that was a bisector of the line, and indeed that the line drawn was a division that actually was a two timing bisector. What justified this presumption?

I never questioned it in my study of Euclid's geometry in high school. But it was out of this original presumption that we were able to construct all sorts of triangles, quadrilaterals and numerous regular and irregular polygons, all too many types to count that we found that we were capable of inscribing and circumscribing figures around circles. And then when we moved into solid geometry later and constructed tetrahedrons inscribed and circumscribed with cubes and spheres, the difficulty of representing such three dimensional figures in two dimensional space strained our ability to study all the different ratios and proportions that were becoming all too complicated to study visually using our two dimensional imaginations.

What struck me in this early study of geometry was the difficulty of representing three dimensional space on a two dimensional plane. There is something planar and level flat in our depictions of complexity in our visual senses. But on the other hand there is something in this reduction of complexity to two dimensions on a flat surface that distorts the way we see accurately multiple dimensions that are full of complexity. This reduction of what we see onto a flat surface distorts the complexity of the phenomena that we wish to depict. In other words it leads to an oversimplification of what we want to depict. It is for this reason that maps are always full of distortions of what we want to display graphically on a flat surface.

How did we know that a right angle has 90 degrees and that a straight line at a point has sides of an angle equally to 180 degrees? How could an angle be straight and still be called an angle, that is, a strait angle? In ordinary ways of speaking there is nothing angular about a straight line. Our ordinary language ways of describing these relationships in geometry were moving away from the conclusions that we were arriving at from our usage of the terms used in our vernacular language. Instead of axiomatizing and finding simpler axioms as mathematicians usually do to define and demonstrate these relationships, it occurred to me that the very language of Euclidean geometry and its axioms and postulates grew out of ordinary ways of speaking as the 
ancient mathematicians confronted what they were seeing and doing in drawing and creating their new constructions in geometry.

Having experienced the problems of gunnery and sighting especially on a bomb sights, it struck me that as human beings we have trouble seeing straight in having two eyes, and we need to close one eye to determine a line of sight. But how do we know that lining up a gun sight or using a singular tubular telescope confirms that a line of sight is a straight line. What seems to be the opposite is that what we do in lining up in sighting is that we define in sighting what it is to be a straight line by definition as one does in surveying.

What I have been doing in my analysis of orientation is to illustrate how we use ordinary language to create the very foundations of our mathematics. Thus, if we are looking for the foundations of mathematical systems, I suggest that we can find first their basis in ordinary vernacular language usage, which itself is in part an already socially constructed set of usages that we ordinarily use to begin to talk about the formal constructions of logic and the mathematics. My suggestion is that our sense of measurement, counting, and ordering is derived from an innate sense of graphical and pictorial representations that has grown with the biological evolution of our culture.

In a way then the language we used in the creation of our mathematical systems is language already developed with the emergence of language in contexts of communication where language changes, and the terms and their uses are socially and politically constructed in specific and different communities. Much of our language that we use every day has in a reversal emerged out of the technical language that has been constructed by political acceptance as standardized technical vocabulary. As our linguistic skills develop, we find a slow evolving change in ordinary language that is changing with new developing technically defined vocabularies coming from the sciences and technologies

We can see how in the process of digitalization how our sense of numbers developed and how in ordinary ways counting developed. And in so far as we see the basis of what is a straight line in terms of vision and alignment, we can also see that we can use our unit of measurement of a line by constantly extending that line of division by replicating segments of that line in either direction by using a divider to extend it further and further with no end in sight. But in the way we act in the ordinary world we are already limited. We find that our line of sight only extends so far within the limits of our visions. What is practical in sighting is that sighting defines our orientation with things that block our line of sight. We see mountains in the distance, or we face the sides of hills or the trees that can even block our view on the slopes of those hills.

In everyday life we orient ourselves with the reference points that we know to exist in our external environment. Note how often then local directions are determined by mountain peaks. And note that surveyors use fixed points with iron stakes that establish boundary lines, but they also need in addition to make their surveys accurate 
in orientation or direction from these fixed points. It is by only knowing the location of those iron stakes that are buried in the ground that surveyors establish the perimeters of your property so as to define the extent of your property rights within the law. Your property lines have local orientations that have been locally constructed.

Modeling orientation is thus a social construction as illustrated in Figure 13.5. Note that orientation relates to how we rotate. Note that one complete rotation is to turn around on a point to face again a line of sight from which we originally turned especially as we do it as customary by continuously turning to your right. Note the very meaning of what it is to be a right angle. It is simply a turn in the direction of your right arm. You do not however completely rotate if you just face to the right. To make a complete rotation you keep making other turns to your right until you face again the line of sight from which you started. You have made 4 right turns in all. Thus, orientation in ordinary ways of speaking of it is how we rotate ourselves in a bodily sense to align our self with our bodily sense of what extends out from our right shoulder and arm. A right angle then is a bodily turn to what extends out from our right hand.

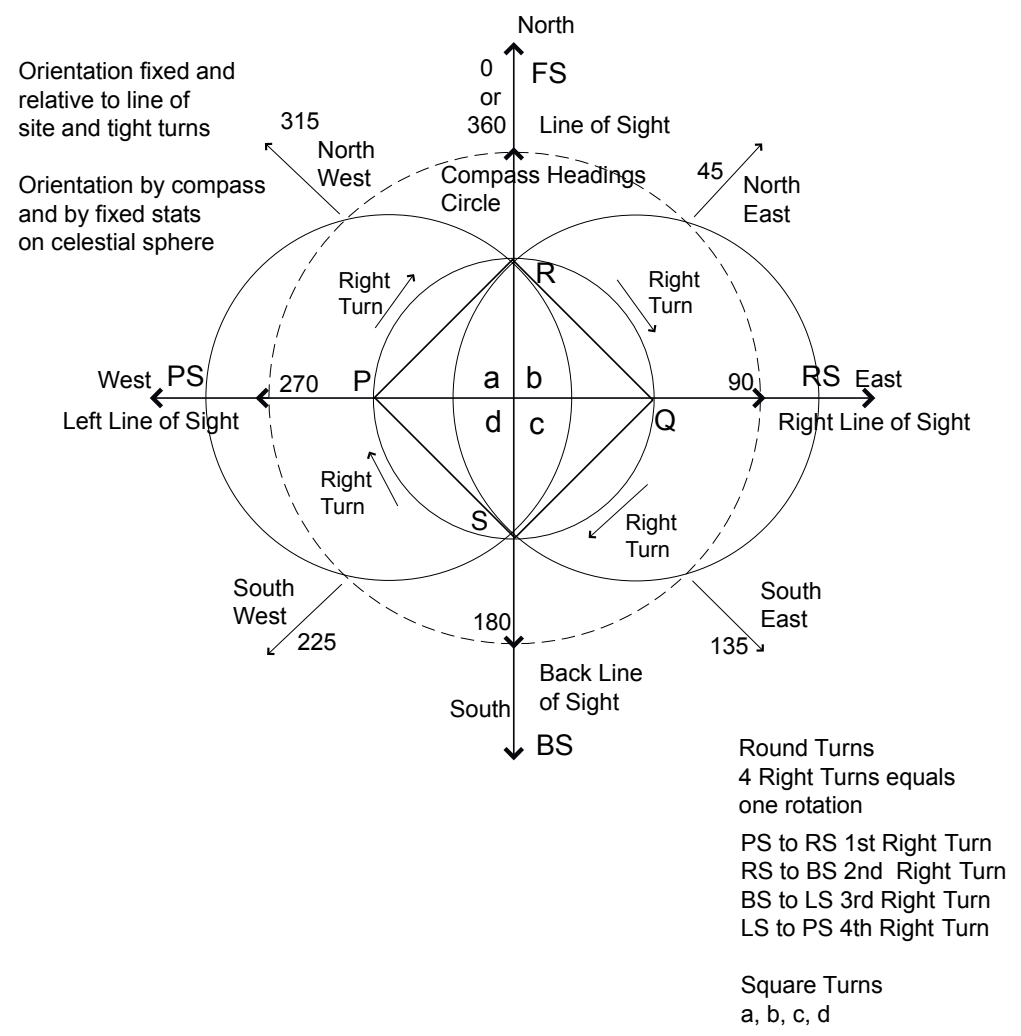

Figure 13.5: Modeling Orientation as a Social Construction 
Note as already discussed to say that a right angle has 90 degrees is a social convention on how to count fractions or increments of turns to the right in fractions of 1/90 of a right turn, which becomes a definition of a unit or a degree of an angular

scale that is $\frac{1}{360}$ of a complete rotation of the body. Such a scale developed by the Babylonians which, as previously mentioned, is thus a more precise measurement in our talking about directions. In other words our 4 turns to the right is a complete rotation, and when these rotations are based upon a 360 degree scale as illustrated a rotation is $4 \times 90$, which is the scale of measurement of the degrees in a circle. Thus the 360 degrees of compass headings is defined by a social convention.

Note the four radii of the circle define and determine the length of the intersecting arcs. They are the four directions on a compass, north, east, south, and west which also can be bisected and subdivided again in eight different nominal directions.

$$
(0,45,90,135,180,225,270,315 \text {, and } 360 \text { degrees })
$$

or

(north, northeast, east, south east, south, south west, west, northwest, and north again)

Note the difference between the compass headings in degrees and compass headings that are directional with their orientation with the fixed stars. In the air corps on B-29's we had a gyro compass on the flight panel to maintain a straight heading, which was zeroed to measure any angular drift off course. On the other hand, we had a flux gate compass that was calibrated to give celestial headings. The difference between the gyro compass and the flux gate compass was that the gyro compass being a compass whose orientation was toward our line of sight, which to maintain a straight course was straight ahead, while the flux gate compass's orientation was towards true geodesic north. One gave a zero heading. The other indicated the compass heading in relation to the angular difference between our line of sight heading and the heading as related to true north. We could then change this geodesic heading of orientation to a true heading on the radar screen by switching our radar returns to be in phase with the fluxgate compass to give us an image pattern that corresponded to the patterns modeled on maps whose orientation was graphically displayed in relationship to true north.

Note we orient ourselves by saying that something is to the right of something, or saying something about its orientation to north or south or east or west. One orients by sight relationships, and we can also orient ourselves by our knowledge of the location of things on a directional grid determined by landmarks, which one presumes when one says such things as, 'It is located just north of the courthouse.'

The practical way of maintaining planar stability in an air plane is to have the wings on a level horizon. But that is impractical to see when in confined places where there are no visible horizons. Other instrumentation based on levels is needed such as an artificial horizon and a needle and ball. On the ground on which we stand the 
standard tool for deciding what is up and what is on a level plane is the use of a plumb bob and a carpenter's level. Plumb bobs are based on the assumption that weight makes us all like Humpty Dumpty subject to tipping over and falling down. Without going into the complications of gravity and sticking to our ways of describing what is up and down and what is on a level, we need to see that all these relationships and complications depend on notions of standing in a fixed and in a stable frame.

But note how a level seems to work. The basic common assumption about liquid is that it seeks its own level which is defined by the boundaries or sides of its container. We have no problem with water in containers on ships at sea of knowing what it is in being flat. From the point of the ship on the sea, it is assumed that the sea is level and extends to the horizons. But now we know that is not true. The sea level is a surface on the earth's sphere, so it is spherical. But when we are in a house and want to determine if a beam or a floor is level we need a device or tool called a level. We use it based upon the presumption that a liquid seeks its own level when contained on a level flat plane such as on a lake or on the water behind a dam.

Of course a level has a bubble in a liquid, and it has a midpoint to line up the level with the floating bubble. Instead of thinking that the bubble moves and wants to determine, what it is to be level by some sort of Spinazoan conatus of liquids in seeking their own level, we need to think of a liquid trying to enter into the space that eliminates the bubble. It is not the bubble that rises when we tilt a level, but it is the hydraulic pressure of the liquid trying to occupy the space of the bubble, which it cannot do. It is the hydraulic pressure on each of side of the bubble that seeks a stable equilibrium between the liquid that is exerting pressure on both sides of the bubble. When those two forces are in equilibrium, the bubble is caught between two equal hydraulic pressures created when the liquid is level on the surface upon which the level lies.

Note again what it is to be up and to be down again relates to how our bodies relate to their environment. Up and down depend on how we orient and frame ourselves within terms of our ordinary language. It depends on the everyday forms of speech that has been developed in the evolution of language in our everyday culture in talking about how we relate to each other and how we relate to our environment in the contexts within which we live. Measurements are ways we have of segmenting both our orientations and our linear perspectives. And units of measurement are agreed upon ways of making those divisions so as to be precise about how we act in reference to them in finding ourselves standing in an upright place on this earth.

Note too that the geometry of circles and ellipses as well as parabolas and hyperbolas are divided by the same measurable units the same way that we divide lines, planes, and three dimensional spaces. And those divisions are described ultimately in the language of our vernacular that describes the very methods of their generation. We generate our standardized terms of measurement from the way they have emerged in our describing our stand point of vision in looking at the world. It is language that has emerged from the evolutionary development of language from 
biological origins. We are upright creatures standing tall. As D. H. Lawrence expressed it, 'We ought to dance with rapture that we should be alive and in the flesh, and part of the living cosmos. That I am part of the earth my feet know perfectly.' Such is our state of equilibrium in being a stabilized point within it. We need to orient ourselves in these elemental formal frames to aid us in maintaining our upright equilibrium. We need to develop these simple frames to construct how we look and visualize the world that we live in and of which we are a part. We do it to show ourselves the way to function in it and to exist so as to be a part of it. We are in it, of it and a part of it. 